\title{
A Rare Case of Posterior Mediastinal Seminoma Mimicking Primary Lung Neoplasm
}

\author{
Sian Yik Lim MD, Grerk Sutamtewagul MD, Ragesh Panikkath MD, Fred Hardwicke MD
}

\begin{abstract}
We report an atypical case of posterior mediastinal seminoma. Mediastinal seminomas are a rare form of tumor usually located in the anterior mediastinum. Our case presented as a diagnostic challenge because of the difficulty of differentiating the primary mediastinal mass from a primary lung neoplasm. Our case highlights the fact that seminomas may occur in the posterior mediastinum and the importance of considering a broad differential diagnosis, especially in cases of poorly differentiated carcinoma of the mediastinum.
\end{abstract}

Key words: primary mediastinal seminoma, posterior mediastinum, primary lung neoplasm, immunohistochemical staining, tissue biopsy

\section{INTRODUCTION}

Mediastinal seminomas are a rare form of tumor, accounting for three to four percent of mediastinal masses. ${ }^{1}$ Their usual location is in the anterior mediastinum. We report an atypical case of primary mediastinal seminoma occurring in the posterior mediastinum in a 51-year-old man. Posterior mediastinum seminomas are extremely rare with only a few cases previously reported. ${ }^{2,3}$ Our case is interesting because it was difficult to differentiate the posterior mediastinal mass from a primary lung tumor due to extension of the mass into the upper and lower lobes of the lung. This feature has not been mentioned in the other case reports. In cases of undifferentiated carcinoma of the mediastinum, additional evaluation of tissue biopsy using immunohistochemical stains is important.

Corresponding author: Sian Yik Lim MD

Contact Information: sianyik.lim@ttuhsc.edu DOI: 10.12746/swrccc2013.0102.023

\section{Case Presenttion}

Our patient is a 51-year-old previously healthy man with significant smoking history (50 pack years) who presented to our hospital with a chief complaint of a three week history of hemoptysis. He began to notice a productive cough with greenish sputum three months prior to presentation. This was associated with bilateral posterior pleuritic chest pain and a 40 pound weight loss over two months. His cough progressively worsened, and he began to develop hemoptysis. He was initially treated with a 10 day course of doxycycline with no improvement of his symptoms. A chest $X$-ray (Figure 1) showed a mass in the right lung which led to referral to our hospital. On presentation, he had right sided wheezes on physical exam. Computed tomography (CT) scan of the chestshowed an irregular poorly enhancing mass in the right perihilar region extending into the right upper and lower lobes with encasement of the right bronchus intermedius (Figure 2). An ultrasound-guided transbronchial needle biopsy of the mass revealed a poorly differentiated tumor consisting of large cells with vesicular irregular nuclei and abundant necrosis. Additional staining 


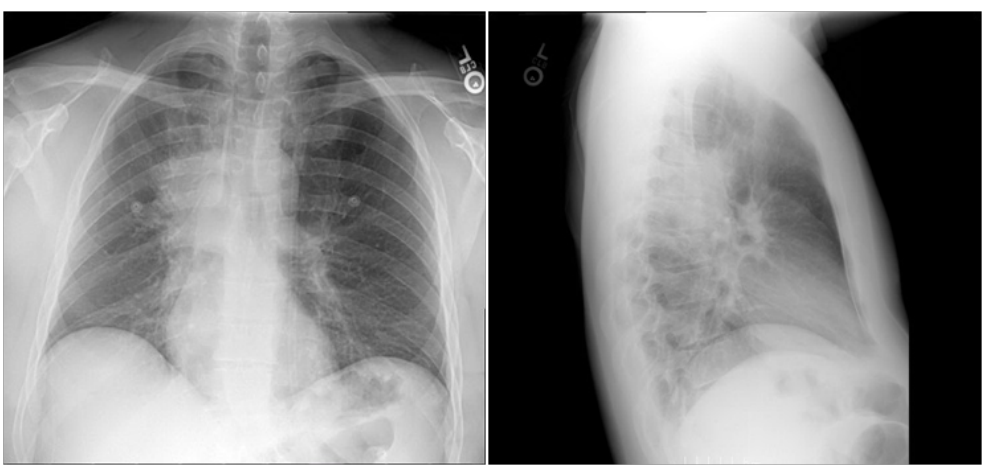

Figure 1. Chest X-ray on admission, showing a mass in the right lung.

showed an immunophenotype positive for cytokeratin (AE/AE3) and placental alkaline phosphatase and negative for CK6, CK20, TTF1, p63, and CD30. This pattern was consistent with the pathological diagnosis of seminoma. Scrotal ultrasound did not demonstrate any mass present. The tumor markers beta human chorionic gonadotropin (beta-HCG), lactate dehydrogenase, and alpha fetoprotein (AFP) were negative. Physical exam and CT scans of the head, abdomen, and pelvis did not reveal other foci of metastasis. He was diagnosed with primary mediastinal seminoma and was started on bleomycin, etoposide, and cisplatin. His symptoms improved significantly after his first cycle of chemotherapy, and he remains on treatment.

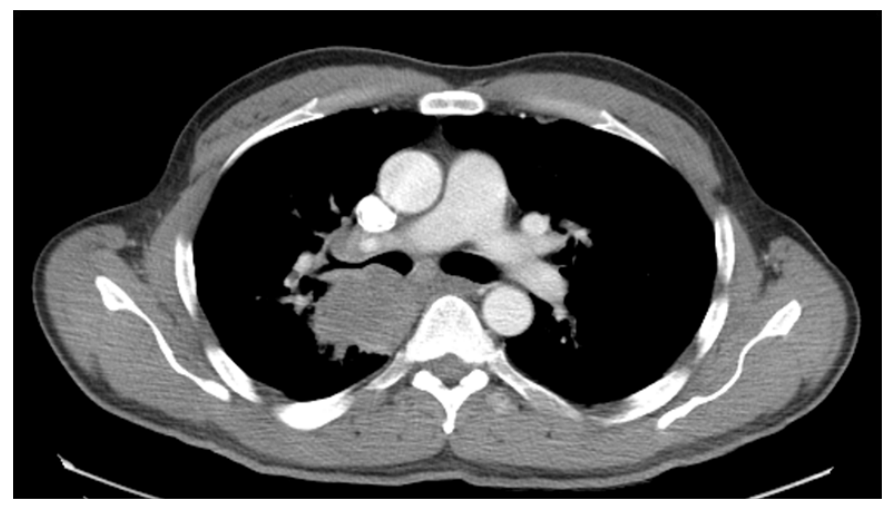

Figure 2. CT scan showing a right mass extending from right perihilar region.

\section{Discussion}

Germ cell tumors are classified as extragonadal if there is no evidence of a primary tumor in the gonads. Extragonadal germ cell tumors typically arise in midline locations, and in adults the most common sites are the anterior mediastinum, retroperitoneum, pineal, and suprasellar regions. Posterior mediastinal seminoma is a rare occurrence with only a few cases reported in the literature. ${ }^{2,3}$ Our case was atypical because the tumor extended into the lung, and this led to an initial impression of a primary lung neoplasm.

The gonad emerges on the ventromedial surface of the mesonephros at the fourth week of gestation. Primordial germ cells migrate from the wall of the yolk sac along the dorsal mesentery of the hind gut to the gonadal ridges. Classically, it is thought that extragonadal germ cell tumors arise from primordial germ cells 'misplaced' due to aberrant migration during embryogenesis. However, this hypothesis has been challenged, and reverse migration of carcinoma in situ lesions in the testis has been suggested as an alternative hypothesis. ${ }^{4}$

Primary mediastinal seminomas cause onethird of malignant mediastinal germ cell tumors and occur predominantly in men age 20 to 40 year of age. The morphologic features of primary mediastinal seminoma are similar to those tumors occurring in the gonads. ${ }^{5}$ As in gonadal germ cell tumors, they occur more commonly in young adults, have i (12p) karyotypic abnormalities, and are sensitive to carboplatin based chemotherapy regimens. About 20\%-30\% of patients are asymptomatic at the time of presentation. ${ }^{6}$ When symptoms are present, they relate to the size and location of the tumor which can compress and/or invade mediastinal structures. The most common presenting symptoms of mediastinal seminoma include chest pain (39\%), dyspnea (29\%), cough (22\%), and weight loss (19\%).

Mediastinal seminomas appear as large, unencapsulated, well-circumscribed masses. On CT scans, seminomas appear large and coarsely lobu- 
lated and typically have homogeneous attenuation equal to that of soft tissue. Calcification is usually absent. These masses can compress mediastinal structures. ${ }^{8}$ As is the case with extragonadal nonseminomatous germ cell tumors, extragonadal seminomas may present as histologically poorly differentiated carcinoma and should be considered in the differential diagnosis of poorly differentiated cancer. ${ }^{2}$ The diagnosis of mediastinal germ cell tumors can be established with a high degree of accuracy through fine needle aspiration biopsy with immunohistochemical analysis. ${ }^{9}$ After the diagnosis of a germ cell tumor is made, a primary testicular tumor must be excluded, and ultrasonography should be performed in all patients since testicular palpation is insufficient to exclude a primary testicular tumor. ${ }^{10}$ Patients with extragonadal seminomas may have small increases in beta-HCG levels. The presence of AFP excludes the diagnosis of a pure seminoma. ${ }^{10}$

Primary mediastinal seminomas are exquisitely sensitive to both cisplatin-based chemotherapy and radiation therapy. Patients such as ours who do not have evidence of nonpulmonary visceral metastases have good prognoses, and these tumors are classified as good risk germ cell tumors by the International Germ Cell Consensus Classification. ${ }^{11}$ Most centers prefer chemotherapy to radiation therapy for patients with primary mediastinal seminoma because of the concern about increased risk of cardiovascular events following mediastinal radiation therapy. Although no randomized controlled trials have been performed, initial chemotherapy was associated with better long-term disease-free survival., ${ }^{1,12}$ There is usually no role for surgical resection/debulking in the initial management of primary mediastinal seminoma. Residual masses post-chemotherapy less than three $\mathrm{cm}$ in size are managed with routine surveillance. If the residual mass is more than three $\mathrm{cm}$, management is controversial since $30 \%$ of these patients have residual tumor. Treatment options include surveillance, additional evaluation with positron emission tomography (PET) scans, or surgical resection.

In summary, this atypical case of posterior mediastinal seminoma highlights the importance of considering these rare tumors in the differential diagnosis of poorly differentiated carcinoma of the mediastinum. Although typically presenting as an anterior mediastinal mass, primary mediastinal seminoma may present in the posterior mediastinum and may be difficult to differentiate from a lung mass. Ordering appropriate tests is important and should lead to an accurate diagnosis and appropriate management.

\section{KEYPOINTS}

1. Although usually presenting as an anterior mediastinal mass, primary mediastinal seminomas may present as a mass in the posterior mediastinum.

2. Primary mediastinal seminomas need to be considered in the differential diagnosis of poorly differentiated carcinoma of the mediastinum. The diagnosis can be established by the ordering additional immunohistochemical staining.

Author Affiliation: Sian Yik Lim, Grerk Sutamtewagul and Ragesh Panikkath are residents in Internal Medicine at TTUHSC in Lubbock, Texas. Dr Hardwicke is an oncologist in the Department of Internal Medicine at TTUHSC.

Received: 03/13/2013

Accepted: 03/21/2013

Reviewers: Aliakbar Arvandi MD, Kenneth Nugent MD

Published electronically: 04/15/2013

Conflict of Interest Disclosures: None

\section{REFERENCES}

1. Jain KK, Bosl GJ, Bains MS, Whitmore WF, Golbey RB. The treatment of extragonadal seminoma. Journal of clinical oncology : official journal of the American Society of Clinical Oncology 1984;2:820-7. 
2. Ravenel JG, Gordon LL, Block MI, Chaudhary U. Primary posterior mediastinal seminoma. AJR American journal of roentgenology 2004;183:1835-7.

3. Makiyama K, Senga Y. Primary seminoma in the posterior mediastinum. The Journal of urology 2001;165:908.

4. Chaganti RS, Houldsworth J. The cytogenetic theory of the pathogenesis of human adult male germ cell tumors. Review article. APMIS : acta pathologica, microbiologica, et immunologica Scandinavica 1998;106:80-3; discussion 3-4.

5. Moran CA, Suster S, Przygodzki RM, Koss MN. Primary germ cell tumors of the mediastinum: II. Mediastinal seminomas--a clinicopathologic and immunohistochemical study of 120 cases. Cancer 1997;80:691-8.

6. Aygun C, Slawson RG, Bajaj K, Salazar OM. Primary mediastinal seminoma. Urology 1984;23:109-17.

7. Bokemeyer C, Droz JP, Horwich A, et al. Extragonadal seminoma: an international multicenter analysis of prognostic factors and long term treatment outcome. Cancer 2001;91:1394-401.

8. Rosado-de-Christenson ML, Templeton PA, Moran CA. From the archives of the AFIP. Mediastinal germ cell tumors: radiologic and pathologic correlation. Radiographics : a review publication of the Radiological Society of North America, Inc 1992;12:1013-30.

9. Chhieng DC, Lin O, Moran CA, et al. Fine-needle aspiration biopsy of nonteratomatous germ cell tumors of the mediastinum. American journal of clinical pathology 2002;118:418-24.

10. Gilligan TD, Kantoff PW. Extragonadal germ cell tumors involving the mediastinum and retroperitoneumWaltham, MA: UpToDate; 2013.

11. International Germ Cell Consensus Classification: a prognostic factor-based staging system for metastatic germ cell cancers. International Germ Cell Cancer Collaborative Group. Journal of clinical oncology : official journal of the American Society of Clinical Oncology 1997;15:594-603.

12. Fizazi K, Culine S, Droz JP, et al. Initial management of primary mediastinal seminoma: radiotherapy or cisplatin-based chemotherapy? Eur J Cancer 1998;34:347-52. 